\title{
LONGITUDINAL COUPLING IMPEDANCE OF THE APS STORAGE RING*
}

\author{
Yong-Chul Chae ${ }^{\dagger}$, Katherine Harkay, Xiang Sun \\ Advanced Photon Source, Argonne National Laboratory, Argonne, IL 60439 USA
}

\section{Abstract}

The three-dimensional wake potentials of the APS storage ring have been reconstructed according to the impedance database concept. Every wakefield-generating component in the ring was considered including small-gap insertion device (ID) chambers, rf cavities, shielded bellows, beam position monitors, synchrotron radiation absorbers, scrapers, flags, various chamber transitions, septum chambers, and pulsed kickers. In this paper the result for the longitudinal wake potentials and its impedance are presented. The total impedance is not model-based but the direct sum of numerical functions. The fit parameters for the broadband resonator model are also included as a convenient representation. We used this impedance model to investigate the observed microwave instability, namely bunch lengthening, anomalous energy spread, and coherent excitation at high synchrotron sideband.

\section{IMPEDANCE DATABASE}

The concept of impedance database is described in the companion paper [1]. We report highlights of building the database for the longitudinal impedance.The horizontal and vertical impedance are reported separately $[2,3]$.

\section{$R f$-Sector (rf-cavities and transition)}

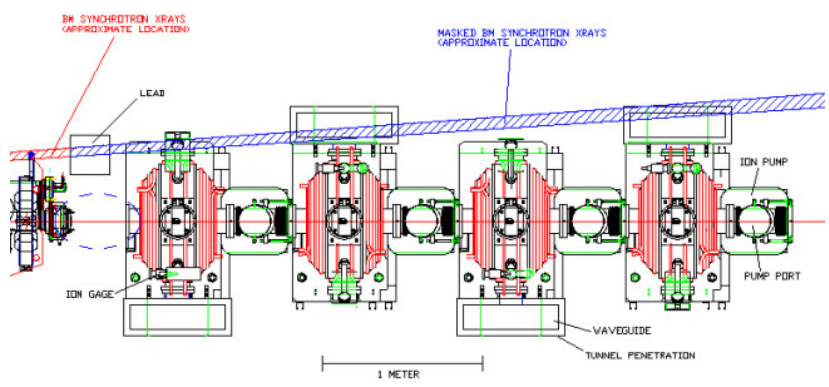

Figure 1: Rf sector showing four cavities.

The rf cavities at the APS storage ring provide energy to the beam with $9.5 \mathrm{MV}$ of gap voltage distributed in sixteen cavities in four sectors. One of the $\mathrm{rf}$ sectors is shown in Fig. 1.

An rf sector consists of four cavities and the transition of vacuum chamber at both ends. The complexity is simplified in the simulation to axially symmetric geometry. We used the program $\mathrm{ABCI}$ in order to calculate wake potential The wake potential of one $\mathrm{rf}$ sector for a $5 \mathrm{~mm}$ bunch is shown in Fig. 2; the cavityonly wake potential is also included for comparison. We

\footnotetext{
* Work supported by the U.S. Department of Energy, Office of Basic Energy Sciences under Contract No. W-31-109-ENG-38.

†chae@aps.anl.gov
}

note that there exists significant interference effect between the transition and cavities.

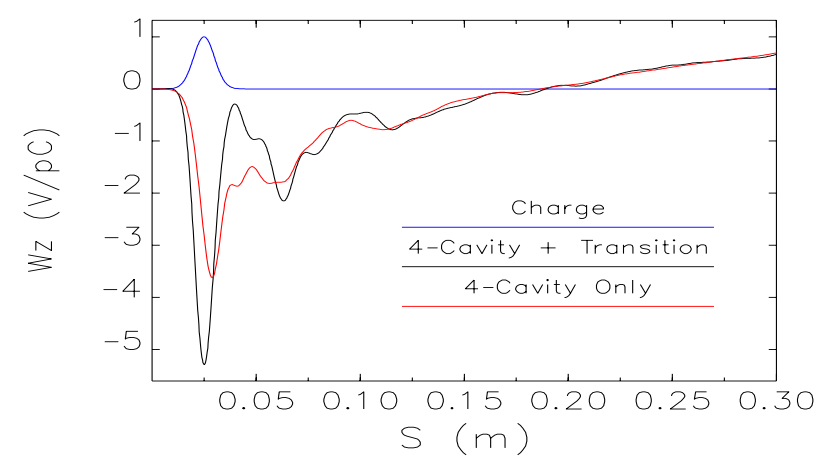

Figure 2: Total wake potential of rf sector. Wake potential of four cavities only is also shown for comparison.
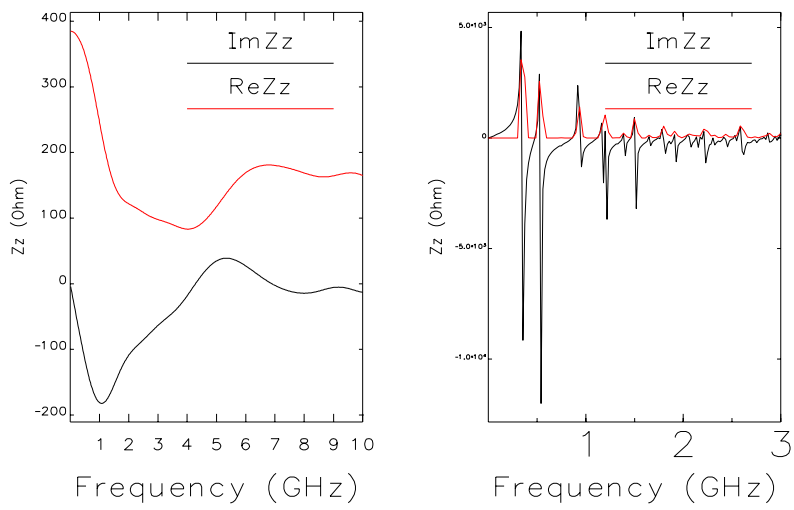

Figure 3: Impedance of the wake over $30 \mathrm{~cm}$ (left); impedance of the wake over $10 \mathrm{~m}$ (right).

The impedance, the Fourier transform of the wake potential divided by the bunch spectrum, is shown in Fig. 3 . The graph on the left shows the impedance of shortranged wake over $30 \mathrm{~cm}$, which shows the characteristic cavity-type impedance in the form of $(1-j) / \sqrt{\omega}$. This short-ranged wake potential is included in the total wake potential. Note the imaginary part of the impedance is negative.

The impedance of long-ranged wake over $10 \mathrm{~m}$ is also shown in Fig. 3 on the right. Higher-order modes (HOMs) as well as the fundamental mode resonating at $352 \mathrm{MHz}$ are clearly shown. The total HOM contribution to $\mathrm{Z} / \mathrm{n}$ is $0.2 \Omega$, which was estimated by using the formula

$$
(Z / n)_{\text {ном }}=\sum_{j=2}\left(R_{s} / Q / n\right)_{j},
$$

where $R_{s}$ is the shunt impedance, $Q$ is the quality factor, and $\mathrm{n}$ is the harmonic number. This $(\mathrm{Z} / \mathrm{n})_{\text {ном }}$ is included in the impedance breakdown for $\mathrm{Z} / \mathrm{n}$. 


\section{Synchrotron Radiation Absorber}

In the APS storage ring there are 200 absorbers of five types; one of them, C2 type, is in a crotch shape that intrudes into the vacuum chamber. The schematic used in the simulation is shown on the top of Fig. 4. The deviceunder-test (DUT) depicted on the top requires the "DIRECT" method in the MAFIA simulation whose result depends on the chamber length. Accurate simulation could require a length that is often prohibitively long.

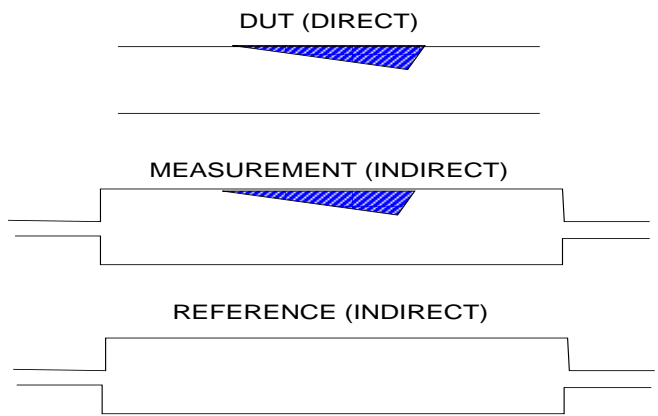

Figure 4: DUT $=$ MEASUREMENT - REFERENCE.

In order to overcome this constraint we used a technique similar to the coaxial wire method, which can be described as DUT=MEASUREMENT-REFERENCE as shown in Fig. 4. The reference chamber is necessary in order to make the simulation geometry suitable for the "INDIRECT" method, whose result doesn't depend on the chamber length and is less susceptible to numerical noise.

The improved result, derived by comparing the new method with the "DIRECT" method, is shown in Fig. 5. The oscillatory wake after the bunch, which could be misread as a "trapped mode," disappeared when a proper simulation technique was applied to the problem.

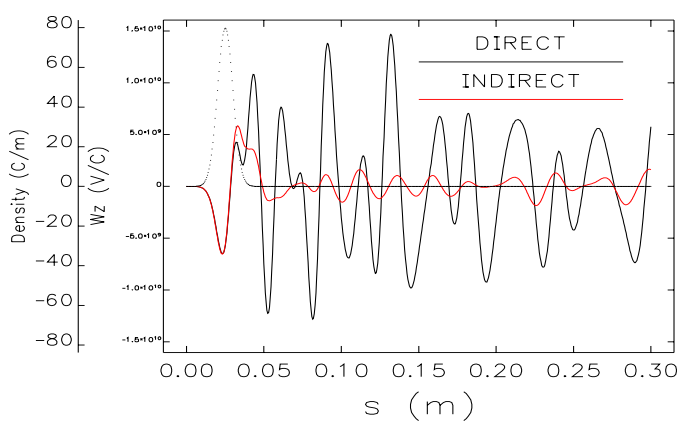

Figure 5: Wake potentials comparing the DIRECT and INDIRECT methods.

\section{TOTAL IMPEDANCE}

The total wake potential of the ring and its impedance are shown in Fig. 6. The important parameter $\mathrm{Z} / \mathrm{n}$ is about $0.22 \Omega$, which is constant up to $22 \mathrm{GHz}$. The breakdown of $\mathrm{Z} / \mathrm{n}$ is presented in Fig. 7, where the rf sector dominates by its $0.2 \Omega$ impedance due to the HOMs.
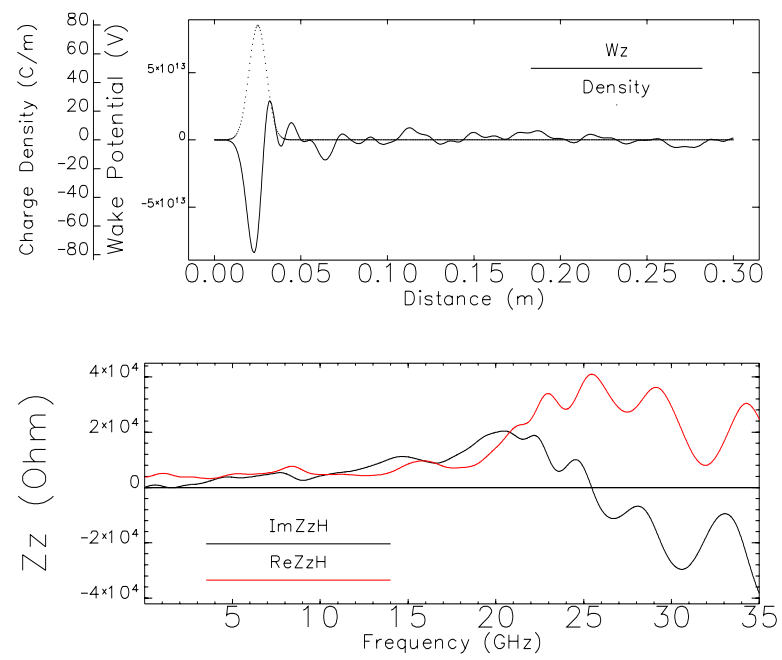

Figure 6: Longitudinal wake potential and impedance of the ring.

\section{Longitudinal Impedance (Ohm)}

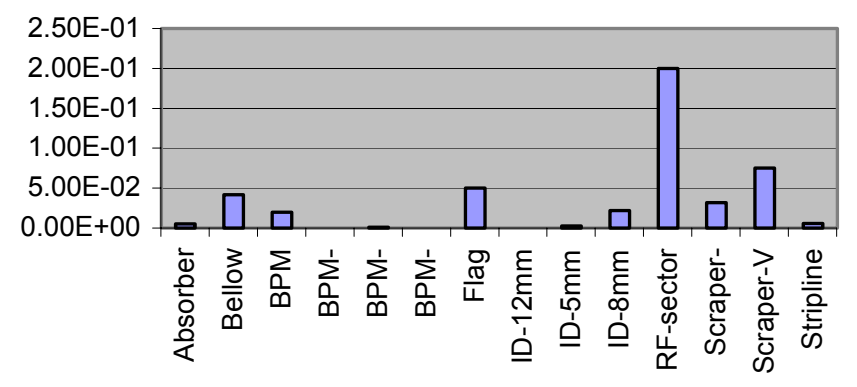

Figure 7: Longitudinal impedance breakdown.

\section{APPLICATION}

The longitudinal microwave instability in the APS storage ring had been measured and reported [4]. As an application of the impedance database we used the total impedance in Fig. 6 as the input to the particle-tracking program elegant [5] in order to reproduce the measured results.

In the simulation we tracked 10,000 particles over 10,000 turns. At each turn the parameters of beam distributions were calculated; two of them were bunch length and energy spread. The average and rms value over 5000 turns for each current level were calculated and their results are shown in Fig. 8. The bunch length (black curve) varies as $\mathrm{I}^{1 / 3}$ over the whole range of current. But the energy spread (red curve) shows a threshold behavior at a current of $6 \mathrm{~mA}$ or $7 \mathrm{~mA}$. This is consistent with the threshold observed experimentally [4]. The rms values depicted as error bars in Fig. 8 also reveal the important information. Above the threshold the rms values increase dramatically, indicating dynamic motion of the bunch in the phase space.

The nature of dynamics above the threshold is revealed by its power spectrum, which is shown in Fig. 9. Each 
trace represents the fast Fourier transform of turn-by-turn bunch length data. The bottom two traces below threshold show the coherent signal at the synchrotron frequencies $f_{s}$ and $2 * \mathrm{f}_{\mathrm{s}}$, representing $\mathrm{m}=1$ and $\mathrm{m}=2$ mode, respectively. However, above the threshold the coherent signal near $4 * \mathrm{f}_{\mathrm{s}}$ is excited; it was also observed experimentally [4].

The simulation using the total impedance reproduced three important aspects of longitudinal phenomena, which are bunch lengthening, energy spread, and synchrotron sideband at $4 * \mathrm{f}_{\mathrm{s}}$. However, this occurred only when the impedance was increased by $80 \%$ of the total impedance, as shown in Fig. 6.

When the maximum frequency of the total impedance was extended to $200 \mathrm{GHz}$ instead of a nominal $100 \mathrm{GHz}$, we observed microbunching of the beam in the simulation as shown in Fig. 10. Apparently buckets formed by the impedance at high frequency trap the particles. We observed that the energy spread increases as the current increases. Unlike the phenomena shown in Figs. 8 and 9, the deformation of bunch was static without a coherent signal at $4 * f_{s}$ or higher. This tantalizing phenomenon is under investigation.

\section{DISCUSSION}

The good agreement between the simulation and the experimental results were obtained by increasing the calculated impedance by $80 \%$. The total inductive impedance at the low frequency expressed as $\mathrm{Z} / \mathrm{n}$ is 0.22 $\Omega$ without ad hoc inclusion of HOM contribution, which is $80 \%$ lower than the estimated value based on the bunch-length measurement [4].

We searched for the missing impedance source to make up for such big differences. We did not miss the impedance source in our impedance database, but perhaps we didn't include the HOM contribution properly in the total impedance.

We show $\mathrm{Z} / \mathrm{n}$ with and without $\mathrm{rf}$-sector contribution in Fig. 11. The rf sector reduces the total impedance in the low frequency range, but the broad and constant $\mathrm{Z} / \mathrm{n}$ didn't change with and without the rf sector, which implies that a HOM contribution of $0.2 \Omega$ is not included in the total impedance. The proper treatment of the $\mathrm{rf}$ cavity might be to subtract the wake potential due to the fundamental mode to make a HOM-only wake potential. Thus pure HOM wake should contribute to the total wake.

\section{REFERENCES}

[1] Y.-C. Chae, "The impedance database and its application to the APS storage ring," these proceedings.

[2] Y.-C. Chae et al., "Horizontal coupling impedance of the APS storage ring," these proceedings.

[3] Y.-C. Chae et al., "Vertical coupling impedance of the APS storage ring," these proceedings.

[4] Y.-C. Chae et al., "Measurement of the longitudinal microwave instability in the APS storage ring," 2001 Part. Accel. Conf., 1817 (2001).
[5] M.Borland, "elegant: A flexible SDDS-compliant for accelerator simulation," Advanced Photon Source Light Source Note 287, 2000.

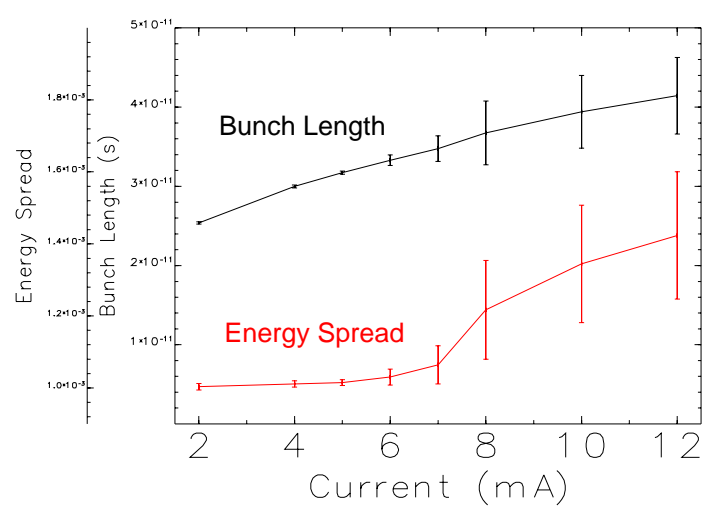

Figure 8: Bunch length and energy spread.

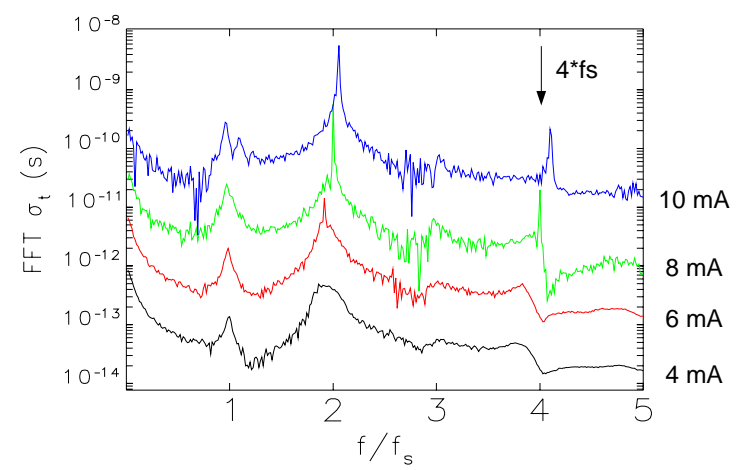

Figure 9: Spectrum of bunch-length oscillation.

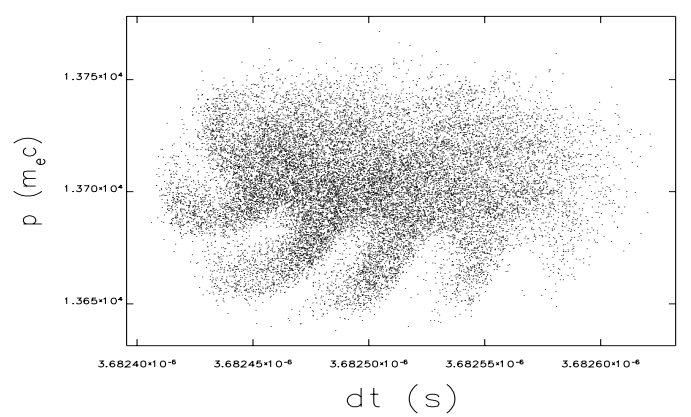

Figure 10: Microbunching of the beam by a highfrequency impedance.

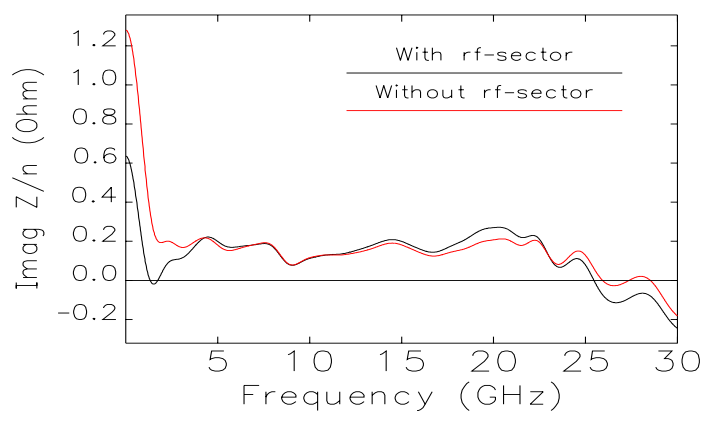

Figure 11: Imaginary part of total $\mathrm{Z} / \mathrm{n}$ with and without $\mathrm{rf}$ sector. 